\title{
Multifetal Pregnancy Reduction
}

\author{
Lt Col SK Rath*, Surg Capt Sushil Kumar+, Lt Col RK Sharma\#, Maj PS Ra**
}

MJAFI 2004; 60 : $67-68$

Key Words : Embryo reduction; Multiple pregnancy

\section{Introduction}

$\mathrm{T}$ he incidence of higher order multiple gestation has undergone a sea change in recent times. Whereas the incidence of triplet is 1 in 64,000 in spontaneous conception, the same occurs in 1 to $3 \%$ of conceptions arising out of assisted reproduction [1]. With increase in the order of multiple pregnancy, the number of pregnancy crossing the period of viability decreases. Most of those who do cross end up in preterm delivery. Thus there is an unacceptable rise in pregnancy wastage and neonatal morbidity with long term sequelae. To address these problems multifetal pregnancy reduction (MFPR) was developed with the aim to improve the poor prognosis of pregnancies with three or more fetuses [2]. It is a therapeutic intervention in which potassium chloride is injected under ultrasonic guidance in to thoracic cage of selected cofetus (es). A case is reported because of uncommonness of the procedure.

\section{Case Report}

A 24 year old primigravida, wife of a serving personnel was transferred from a service hospital to our centre with the diagnosis of quadruplet pregnancy. She was married for 5 years and had conceived after treatment for infertility in the form of ovulation induction and timed intercourse. She had amenorrhoea of 10 weeks at the time of reporting to our centre.

Physical examination revealed an averagely built and nourished patient with no pallor or pedal edema. Systemic

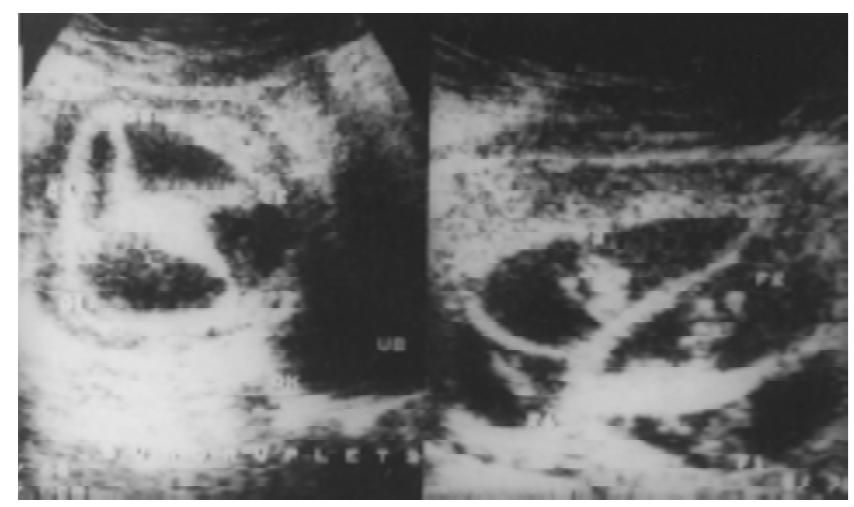

Fig. 1 : Ultrasound scan showing four gestational sacs with fetus examination was normal. Uterine fundal height was 16 weeks size. Ultrasound showed a gravid uterus with four gestational sacs corresponding to the period of amenorrhoea (Fig-1). Cardiac activity was seen in all four. She was planned for fetal reduction. The risks and benefits were explained to the couple and informed consent was taken. Under general anaesthesia in lithotomy position a transvaginal 5 mega hertz ultrasound mounted with a guide plate was introduced. A $250 \mathrm{~mm}$ long 22 gauge needle was passed through the guide plate. Under ultrasound visualization the needle was passed through the vaginal fornix into the uterus and then into the thoracic cage of a fetus situated away from the internal os. Potassium chloride $(0.4 \mathrm{ml})$ was introduced into the fetal thoracic cage. Cardiac asystole was immediately seen. After the cardiac activity was confirmed to have ceased for two minutes the procedure was repeated for one more fetus. The probe with needle was removed and absence of any significant vaginal bleeding was confirmed at the end of the procedure. Postoperatively she was managed with tocolytics for twenty four hours. Recovery was uneventful. Follow up ultrasound showed normal cardiac activity in the remaining two fetuses (Fig 2). The patient was subsequently followed up in the referring hospital. She had an uneventful antenatal period till 35 weeks when caesarean section was done for premature rupture of membrane. The neonates are doing well.

\section{Discussion}

Several terms have been used to describe this procedure of reducing the number of fetus in a multiple

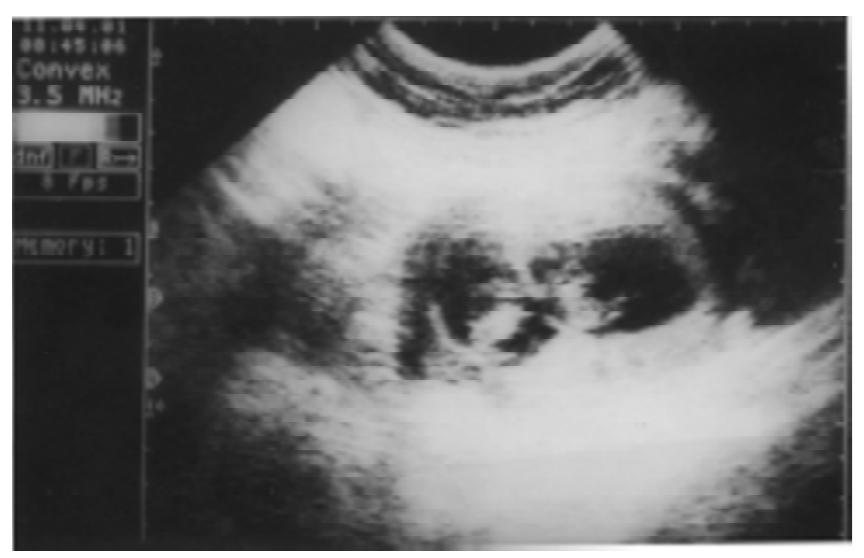

Fig. 2 : Ultrasound scan showing two remaining fetuses three days after MFPR

"Classified Specialist (Obstetrics \& Gynaecology), 151 Base Hospital, C/o 99 APO, ${ }^{+}$Senior Advisor (Obstetrics \& Gynaecology), INHS Asvini, Colaba, Mumbai, ${ }^{\#}$ Classified Specialist (Obstetrics \& Gynaecology), Army Hospital (R\&R), New Delhi - $110010,{ }^{* *}$ Graded Specialist (Obstetrics \& Gynaecology), HQ Imtrat, C/o 99 APO. 
pregnancy. A consensus has been reached to use the term 'selective termination' for a procedure performed because of an abnormality in one fetus and MFPR when the indication is solely fetal number without an apparent fetal defect [3]. Though the first report in 1986 used a trans cervical procedure, high rates of complications soon established trans abdominal and trans vaginal approach as methods of choice [4,5]. The ideal period of intervention is between 9 to 12 weeks. The fetuses to be targeted depends on a number of factors. If any fetus appears to be abnormal, the same is to be selected for termination. If two fetuses appear as monochorionic, then both or neither should be selected. It is also preferable not to choose the embryo closest to the cervix to obviate the chances of ascending infection. Theoretical risk of development of consumptive coagulopathy following MFPR necessitates a follow up with coagulation profile for a short time. However, clinical development of such coagulopathy has not been reported.

Pregnancy losses occurring within a week of fetal reduction are procedure related. For the latter ones, it is not possible to be certain whether or not the loss was procedure related. It is encouraging to note that there has been consistent improvement over the years in pregnancies going close to term and delivering neonates who are free from any significant morbidity following MFPR. Largest database on the subject compiled by Evans quotes the losses to be $12 \%$ for theperiod 1995 2000 [6]. Though there is plenty of data to support the reduction from triplets to twin purely on a risk benefit analysis, the same cannot be said for reducing twins to singleton.

MPFR has evolved as an adjunct to infertility therapy and is not to be viewed as an end in itself. Availability of facility to cryopreserve embryo, research by reproductive specialists and regulation by professional bodies is likely to bring down the incidence of higher order pregnancy and hence the need of MFPR. However, so long as the need is there, it will be undertaken with respect for all medical and ethical issues.

\section{References}

1. Levene MI, Wild J, Steer P. Higher multiple births and the modern management of infertility. Br J Obstet Gynecol 1992;99:607-10.

2. Berkowitz RL, Lynch L, Chitkara U, Wilkins IA. Selective reduction of multifetal pregnancies in the first trimester : $\mathrm{N}$ Engl J Med 1988;318:1043-6.

3. Dumez Y, Oury JF. Method for first trimester selective abortion in multiple pregnancy. Contrib Gynecol Obstet 1986;15:50-4.

4. Illan E, Timor T, David BP. Multifetal pregnancy reduction by trans vaginal puncture evaluation of technique used in 134 cases. Am J Obstet Gynecol 1993;169:799-809.

5. Evans MI, Littman L, King M, Fletcher JC. International collaborative experience in 1798 patients having multifetal pregnancy reduction : a plateauing of risks and outcomes. J Soc Gynecol Invest 1993;3:23-8.

6. Evans MI, Berkowitz RI, Waapner RJ, Carpenter RJ, Goldberg $\mathrm{JD}$, Ayoub MA. Improvement in outcomes of multifetal pregnancy reduction with increased experience. Am J Obstet Gynecol 2001;184(2):97-110.

Patient: I always see spots before my eyes.

Doctor: Didn't the new glasses help?

Patient: Sure, now I see the spots much clearer. 